\title{
Experimental Study on the Brittle-Ductile Response of a Heterogeneous Soft Coal Rock Mass under Multifactor Coupling
}

\author{
Weijian Yu (i), ${ }^{1,2}$ Genshui Wu $\left(\mathbb{D},{ }^{1}\right.$ Baifu An, ${ }^{2}$ and Ping Wang ${ }^{2}$ \\ ${ }^{1}$ School of Resource \& Environment and Safety Engineering, Hunan University of Science and Technology, Xiangtan, \\ Hunan 411201, China \\ ${ }^{2}$ Hunan Key Laboratory of Safe Mining Techniques of Coal Mines, Hunan University of Science and Technology, Xiangtan, \\ Hunan 411201, China \\ Correspondence should be addressed to Weijian Yu; ywjlah@163.com and Genshui Wu; wu_genshui@163.com
}

Received 27 November 2018; Revised 27 February 2019; Accepted 28 March 2019; Published 2 May 2019

Guest Editor: Bisheng Wu

Copyright (c) 2019 Weijian Yu et al. This is an open access article distributed under the Creative Commons Attribution License, which permits unrestricted use, distribution, and reproduction in any medium, provided the original work is properly cited.

\begin{abstract}
After a gas drainage event causes different degrees of initial porosity in the coal seam, the heterogeneity of the coal mass becomes much more obvious. In this paper, soft coal testing samples with different degrees of heterogeneity were prepared first by a new special experimental research method using hydrogen peroxide in an alkaline medium to generate oxygen. Then, a series of mechanical tests on the soft coal mass samples were carried out under multiple factor coupling conditions of different heterogeneities and confining pressures. The results show that with a low strength, the ductility failure characteristic and a kind of rheology similar to that for soft rock flow were reflected for the soft coal; i.e., the stress-strain curve of the coal mass had no apparent peak strain and residual strength. An interesting phenomenon was found in the test process: there was an upwardly convex critical phase, called the brittle-ductile failure transition critical phase, for the heterogeneous soft coal mass between the initial elastic compression phase and the ductile failure transition phase in the stress-strain curve of the coal mass. An evolution of the brittle-ductile modulus coefficient of the soft coal was developed to analyze the effect of the internal factor (degree of heterogeneity) and external factors (confining pressure) on the transition state of the brittle-ductile failure of soft coal. Further analysis shows that the internal factor (heterogeneity) was also one of the essential factors causing the brittle-ductile transition of soft coal.
\end{abstract}

\section{Introduction}

A soft coal mass with a complex composition and a cementation structure is a typical inhomogeneous medium and has more complicated mechanical properties compared with other rock masses [1-7]. In addition, due to the high number of interformational pores, fractures, and microfractures caused by the occurrence of gas, this kind of gas-filled coal mass was found to be damaged to varying degrees [8-11]. In high gas and deep mines, the "three fields of surrounding rock," i.e., the original rock stress field, the mining stress field, and the support stress field, will change after a gas drainage event or roadway excavation, which will result in instability of the surrounding rocks and even lead to some dynamic destructive disasters, such as coal and gas bursts or rock burst, causing an unrecoverable loss of economy and time
[12-20]. In particular, under a high-stress environment and a complex disturbance stress field, the high-pressure gas inside the coal mass not only weakens the strength of the coal but also leads to nonlinear characteristics of the coal mass. Therefore, the mechanical properties of a heterogeneous coal rock mass are dynamically changing under the influence of different stress fields and gas pressure fields in the deep underground.

For the heterogeneity of the soft coal mass, most scholars in past research programmes believed that the parameter value of the mesoscopic unit of the coal mass followed the Weibull distribution [21-24] which could be evaluated by using one defined Weibull distribution function. Additionally, numerous results show that the macroscopic mechanical properties of the soft coal mass depend largely on the heterogeneity of the mesoscopic parameters $[25,26]$, which is the 
main reason for the macroscopic inhomogeneity and nonlinear characteristics of the coal mass under an external load [27-31]. Feng and Zhao [32] studied the relationship between the macroscopic deformation behaviour of rock and the mechanical properties of the mesoscopic particles and found that regardless of whether its mesoscopic particles are an ideal elastic-plastic material, an extremely brittle material, or a transitional material, the macroscopic deformation behaviour of the rock depends only on its heterogeneity. Furthermore, the more uniform the mesoscopic particle distribution of the rock is, the more obvious the brittle characteristics of the rock. In contrast, the less uniform the mesoscopic particle distribution of the rock is, the more obvious the elastoplastic characteristics of the rock. Yang et al. [33] found that the inhomogeneity caused by material defects played a key role in the distribution of small voids and cracks in the materials, as well as in the mode of crack propagation. Hudyma et al. [34] showed that the relationship between the mechanical characteristics and the porosity of tuff was that with increasing porosity; its compressive strength and elastic modulus were reduced. From the above, heterogeneity is clearly an important index and has been widely used by scholars to evaluate the mechanical parameters of coal and rock masses. Moreover, the damage accumulation of the rock mass depends largely on the homogeneity of the rock, especially the impact fragmentation of brittle heterogeneous solids [35].

Recently, with the increasing depth of mining, the mechanical characteristics of the coal and rock around roadways at high pressure, high gas pressure $[36,37]$, and high temperature [38, 39] have been systematically researched. Yang et al. [40] obtained the failure mechanisms and stability control technology for a deep roadway with some soft rocks in the Xin'an Coal Mine in Gansu Province, China, and one new useful "bolt-cable-mesh-shotcrete+shell"-combined support was proposed to support the ventilation roadway. Yu et al. [41, 42] proved that the fracture expansion of the coal and rock mass caused its permeability to increase, weakening the surrounding rock strength. Then, they proposed a core support technology with a prestressed truss anchor cable to support the roadway.

The mechanical properties of intact hard rocks with high homogeneity will change under certain conditions. Researchers found that under high temperature, high confining pressure, and other conditions, the rock would transition from brittle failure to ductile failure [43-45]. Generally, the heterogeneity of rocks is a concentrated expression of microcracks and rock defects from a microperspective. From a macro perspective, it is the result of the dynamic development and penetration of macrocracks and pores, as well as the coupling of various other influencing factors such as water and gas. This special property of rock is not an idealized assumption of simple and single conditions but is the result of multidimensional, multifactor, and multilevel coupling. Unfortunately, most of the researches on the special characteristics of the brittleductile transition of rock are concentrated on the analysis of mechanical properties of rock by external single factors (e.g., single confining pressure and single temperature), thus neglecting the influence of mutual coupling with internal factors of rock (e.g., heterogeneity, water, gas, and temperature). Therefore, this paper takes the heterogeneous coal seam mining affected by gas or after gas extraction as the engineering background. A series of mechanical tests were carried out on heterogeneous soft coal samples. The influence of the internal factors (heterogeneity) and external factors (confining pressure) on the brittle-ductile failure and mechanical characteristics of the coal mass was analyzed in detail. The brittleness-ductility of the soft coal mass model was established. Then, the change law of the internal structure of the soft coal mass affected by the gas was inferred.

\section{Effect of Gas on the Heterogeneity and Mechanics of Soft Coal Mass}

Similar to loose soil, the gas-filled coal mass is easily destroyed under a complicated stress condition due to its soft structure, low strength, poor deformation resistance capability, and the fact that the internal area contains many weak surfaces and original damage. After gas drainage in the coal seam, the coal mass becomes loose and soft, showing macroscopic heterogeneity and nonlinear characteristics due to the combined disturbed stress field.

The heterogeneity parameters of the coal medium are reflected by the expansion degree of the specimen (i.e., the initial porosity of the different coal samples), as shown in equation (1):

$$
m=n \cdot k+m_{0}
$$

where $m$ is the heterogeneity parameter of the coal mass, $k$ is the rock material constant, $n$ is the porosity (initial porosity) of the coal mass, and $m_{0}$ is the initial heterogeneity parameter of the soft coal mass.

The comprehensive effect of axial compression, confining pressure, and gas pressure on the gas-filled coal mass can be expressed by an abstract macroscopic effective stress, which is an equivalent parameter to describe the deformation process of the coal mass [46]. The effective stress $\sigma^{\prime}$ can be determined [47]:

$$
\sigma^{\prime}=\sigma-P_{g}+(1-n) P_{g}
$$

where $n=\left(m-m_{0}\right) / k$, the rock porosity is related to heterogeneity $m, P_{g}$ is the pore pressure of the gas, and $\sigma$ is the total stress of heterogeneous coal and rock.

Assuming that the failure of heterogeneous soft coal conforms to the Mohr-Coulomb failure criterion, the effective stress failure criterion can be obtained:

$$
\tau^{\prime}=c+\sigma^{\prime} \tan \varphi
$$

where $c$ is the cohesive force and $\varphi$ is the internal friction angle. 
By substituting equation (2) into (3), we can obtain

$$
\tau^{\prime}=c+\left(\sigma-P_{g}\right) \tan \varphi+P_{g}(1-n) \tan \varphi
$$

Let $(1-n) \tan \varphi=\tan \varphi^{\prime}$, where $\varphi^{\prime}$ is the effective internal friction angle, and the value is related to the heterogeneity of the coal and rock mass.

$$
\tau^{\prime}=c+\left(\sigma-P_{g}\right) \tan \varphi+P_{g} \tan \varphi^{\prime}
$$

Equation (5) shows that the effective shear strength of the heterogeneous coal and rock mass is composed of cohesion, one strength caused by the stress variable $\sigma-P_{g}$ and another strength caused by the stress variable $P_{g}$. The strength caused by the stress variable $\sigma-P_{g}$ is related to the angle of internal friction, while the shear strength caused by stress variable $P_{g}$ is related to the effective internal friction angle.

Let $c^{\prime}=c+P_{g} \tan \varphi^{\prime}$, where $c^{\prime}$ is the effective cohesion. Therefore, equation (5) can be simplified as follows:

$$
\tau^{\prime}=c^{\prime}+\left(\sigma-P_{g}\right) \tan \varphi
$$

The stresses $\sigma-P_{g}$ and $\tau$ in the plane are determined by the stress circles of the principal stresses $\sigma_{1}-P_{g}$ and $\sigma_{3}-P_{g}$, as follows:

$$
\sigma_{1}-P_{g}=\frac{1+\sin \varphi}{1-\sin \varphi}\left(\sigma_{3}-P_{g}\right)+\frac{2 c^{\prime} \cos \varphi}{1-\sin \varphi}
$$

According to equation (7), the obtained theoretical curve of heterogeneous coal is shown in Figure 1. The triaxial compressive strength (TCS) of coal is affected by gas pressure and heterogeneity $m$. Under the action of gas, the TCS of the coal sample decreases linearly with the increase of gas pressure and the coal with higher heterogeneity has lower peak strength.

\section{Test Design for a Heterogeneous Soft Coal Specimen}

3.1. Process Flow and Preparation Principle. Due to unfavorable construction conditions and test equipment factors, it is difficult to obtain raw coal samples underground. However, corresponding rock samples could simulate the characteristics of raw coal [48-50]. Niu et al. [51, 52] have done a lot of researches on natural and reconstituted anthracite coals, and the results show that the swelling strain of reconstituted coal is similar to the homogeneous isotropic variety. Therefore, this method is used to produce heterogeneous soft coal specimens. The specimens consisted of anthracite coal ash through a 100 mesh screen (the average diameter is $150 \mu \mathrm{m})$ as the main aggregate and P32.5 ordinary Portland cement as the main cementitious material, and the degree of heterogeneity was controlled by $30 \%$ hydrogen peroxide, which generated oxygen through the disproportionation reaction in the alkaline medium, as shown in the chemical

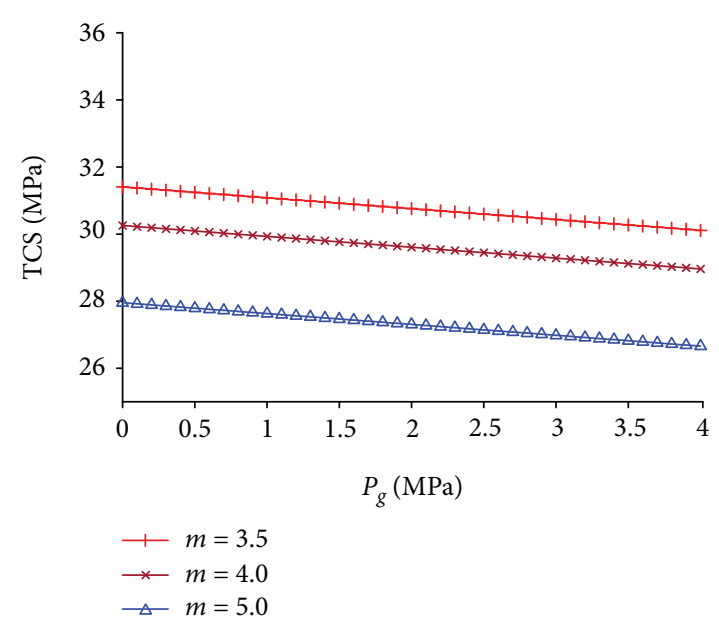

FIgURE 1: Theoretical curves of peak strength, gas pressure, and heterogeneity of heterogeneous soft coal rock mass $\left(\sigma_{3}=2 \mathrm{MPa}\right.$, internal friction angle $=8^{\circ}$ ).

reaction equation (8), to create pores and fissures in the specimens. The basic weight ratio of the white cement to anthracite coal ash in the specimen was $7: 3$, and the water to cement ratio was 0.4 .

$$
2 \mathrm{H}_{2} \mathrm{O}=2 \mathrm{H}_{2} \mathrm{O}+\mathrm{O}_{2} \uparrow
$$

Additionally, to reduce the three-dimensional distribution of pores and cracks in the sample, we first used the mixer to mix and tamp the sample fully. Then, the coal samples that were screened twice by comparing the quality and testing the rock wave velocity were selected for the test.

Figure 2 illustrates the process flow for preparing specimens with different heterogeneities. The main flow of the sample preparation is as follows: First, the raw materials (except hydrogen peroxide) were measured at the set ratio and the uniform slurry was stirred at a water temperature of approximately $20^{\circ} \mathrm{C}$, with the mixing time controlled within $60 \sim 80 \mathrm{~s}$. Then, the designated quantity of hydrogen peroxide was added to the slurry and quickly stirred. The chemical reaction occurred after mixing hydrogen peroxide and other materials. Generally, the basic chemical reaction time and initial setting time of the cement were less than $5 \mathrm{~min}$ and $10 \mathrm{~min}$, respectively. In the preparation of samples, $\mathrm{H}_{2} \mathrm{O}_{2}$ and other aggregates need to be stirred and poured into the mould with other complex procedures. These procedures will take a lot of time, so it is required that adding the $\mathrm{H}_{2} \mathrm{O}_{2}$ mixture should be completed in a relatively short time (controlled within 30-40s). Except for the test specimens into which hydrogen peroxide was not added, the other test samples were filled to $70 \%$ of the mould volume. To reduce error, it was forbidden to cast multiple sets of specimens at once. After the vibration compaction and initial setting of the test specimens, the expansion process was observed and recorded for 8 hours. After removing the expansion and smoothing the ends, the specimens were demoulded from the containers and maintained for $28 \mathrm{~d}$ at room temperature 


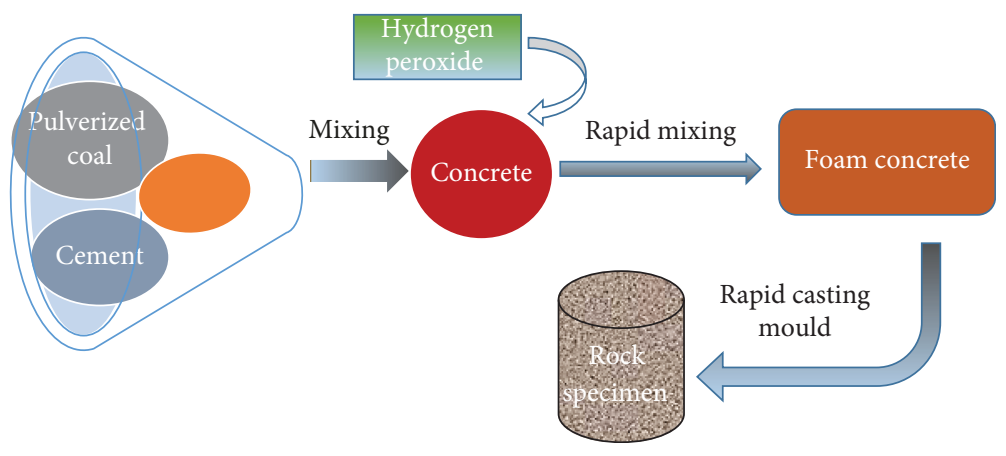

FIgURE 2: Preparation process for a gas-affected heterogeneous soft coal mass specimen.

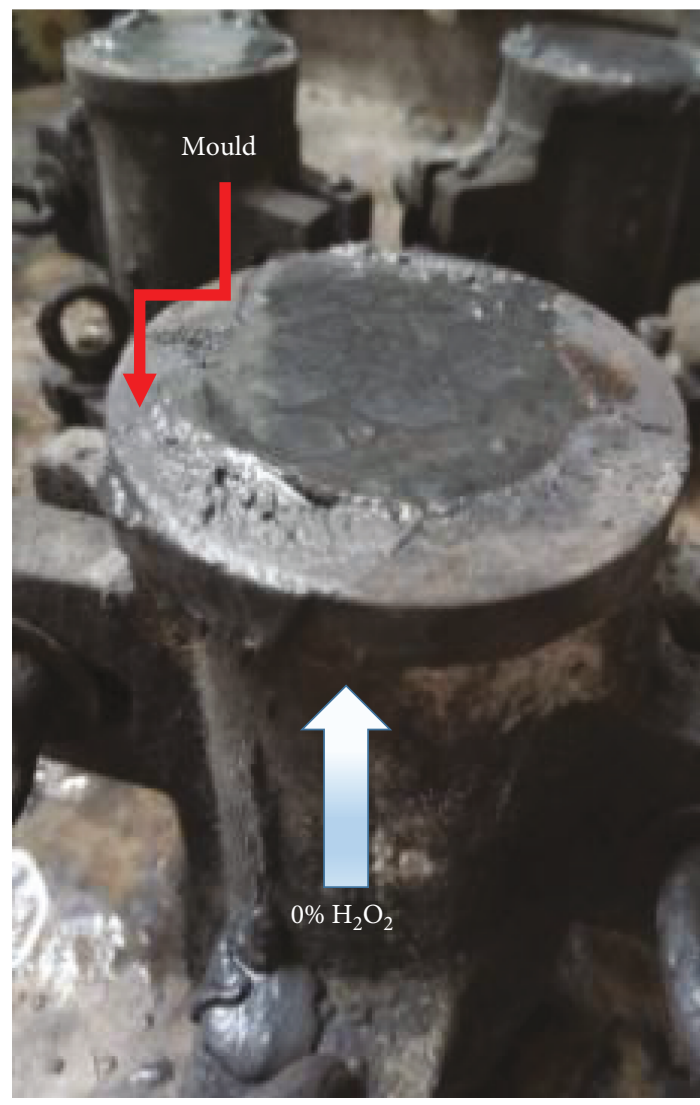

(a)

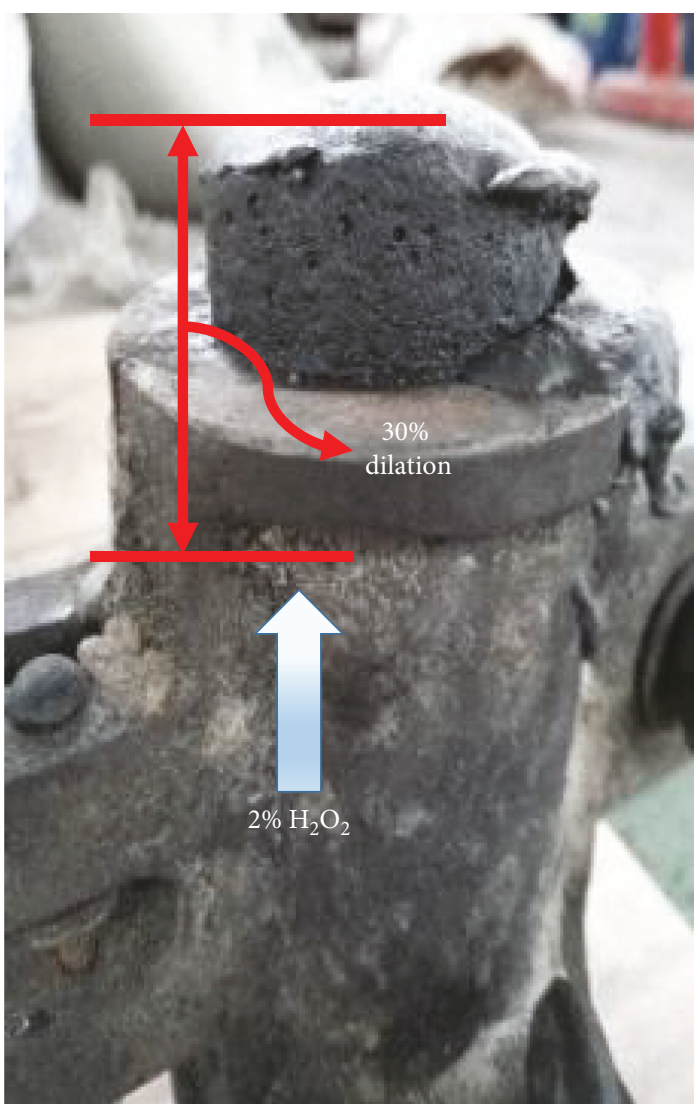

(b)

FIGURE 3: Swelling behaviour of rock-like specimens made with different hydrogen peroxide contents: (a) specimen without expansion $\left(\mathrm{H}_{2} \mathrm{O}_{2}=0 \%, m=1\right)$; (b) specimen with expansion of $30 \%\left(\mathrm{H}_{2} \mathrm{O}_{2}=2 \%, m=4\right)$.

according to the International Society for Rock Mechanics (ISRM) standards [53, 54].

The test moulding tool is a customized three-opening mould made of cast iron, as shown in Figure 3, which can create a standard test piece with a height and diameter ratio of $2: 1(50 \mathrm{~mm} \times 100 \mathrm{~mm})$. Figure $3(\mathrm{a})$ indicates that no expansion occurred in the mould without hydrogen peroxide. The hydrogen peroxide is easily dispersed in the concrete, and the gas velocity and mass can be controlled by the temperature, the concentration of the hydrogen peroxide, and the stirring speed. Different test pieces with different degrees of heterogeneity were made by this process. When only $2 \%$ of hydrogen peroxide was added, the volume of coal expanded by $30 \%$ (Figure 3(b)).

3.2. Test Scheme. The species were all tested in the digital control electrohydraulic servo rock test system developed by the Wuhan Institute of the Chinese Academy of Sciences (RMT150C). The test equipment is mainly used for mechanical property testing of rock and concrete materials. Fourteen sensors are equipped to record the load, stress, strain, displacement, and other parameters from the axial and 
TABLE 1: Relationship between initial porosity and the heterogeneity parameter of the specimen.

\begin{tabular}{lccc}
\hline Number & $\begin{array}{c}\text { Hydrogen peroxide } \\
\text { content }(\%)\end{array}$ & $\begin{array}{c}\text { Average dilation } \\
\text { volume }(\%)\end{array}$ & $\begin{array}{c}\text { Heterogeneity } \\
(\mathrm{m})\end{array}$ \\
\hline A & 0 & 0 & 1.0 \\
B & 1 & 25 & 3.5 \\
C & 2 & 30 & 4.0 \\
D & 3 & 40 & 5.0 \\
E & 4 & 45 & 5.5 \\
F & 5 & 50 & 6.0 \\
\hline
\end{tabular}

transverse direction of the specimen during loading. The settings of the axial loading rate and the confining pressure loading rate are $0.20 \mathrm{kN} / \mathrm{s}$ and $0.05 \mathrm{MPa} / \mathrm{s}$, respectively, and two levels of confining pressure (2.0 MPa and 4.0 MPa) are set for the triaxial test and the uniaxial compression coupling factor test, respectively, for different heterogeneous and gas content soft coal samples.

A comparison test of 6 groups of samples with hydrogen peroxide content varying from $0 \%$ to $5 \%$ (with \% indicating the mass ratio of the hydrogen peroxide content to the aggregate) was established.

According to equation (1), each initial porosity of the soft coal specimen corresponds to the degree of heterogeneity parameter. In this paper, the $k$ value of soft coal is 10 and the value of $m_{0}$ is 1 to reflect the mechanical properties of the heterogeneous soft coal mass. The corresponding relationship is shown in Table 1.

\section{Results and Discussion}

4.1. External Single Factor (Confining Pressure). Figure 4 shows the stress-strain curves of the specimens with heterogeneous $m=1$ under triaxial and uniaxial compression tests. Compared with the uniaxial tests, the test curve obtained under the triaxial confining pressure tests has no apparent peak strain and residual strength, presenting a kind of rheology similar to that for the soft rock flow.

4.2. Internal Multifactor Coupling (Confining Pressure, Heterogeneity, and Gas Content). The triaxial compressive stress-strain test results of the soft coal masses with different heterogeneities and gas content under different confining pressure couplings are shown in Figure 5. The test curve is similar to the experimental results of the coal mass with less homogeneity, and there is no obvious peak strain or residual strength. In the process of compression, the effects of the degree of initial porosity (i.e., the heterogeneity parameter $m$ ) on the coal mass deterioration are different (Figure 5(a)). The microcracks and pores can produce more massive fissures during the stress loading process, and the specimens will be destroyed when the pores and fissures penetrate to the end (Figure 5(b)).

The failure of the specimen (Figure 5(b)) shows that dilatancy failure is the main damage form for the soft coal mass with a low degree of heterogeneity ( $m$ value is within $1 \sim 4)$. With increasing degree of heterogeneity $(m>5)$, the

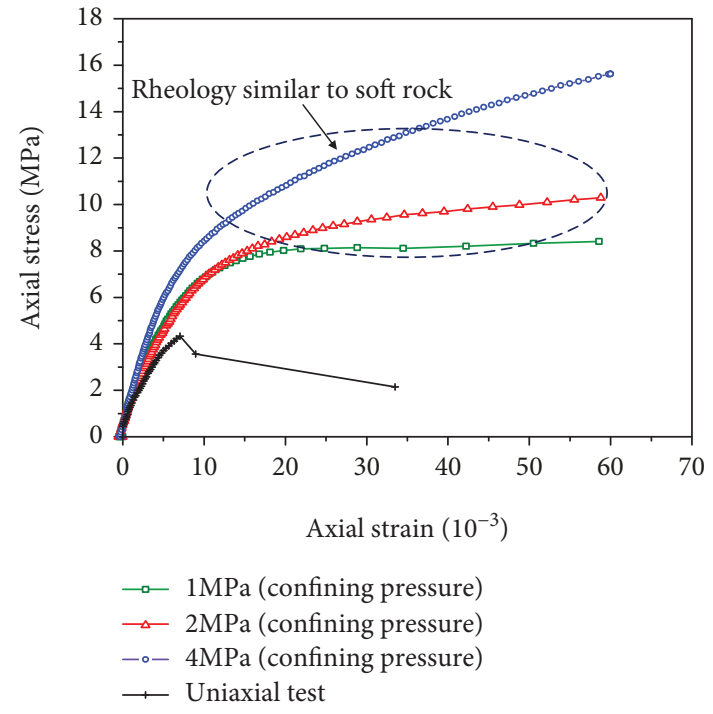

FIgURE 4: Stress-strain curves of triaxial and uniaxial compression tests for nonexpansive specimens $(m=1)$.

distribution of the internal mineral medium becomes more nonuniform, the overall structure becomes worse, and microcracks and pores gradually develop, while the cohesive force decreases slowly. At this point, dilatancy failure can be clearly observed. As the degree of heterogeneity of the coal mass increases, the damaged form of the specimens will become loose and broken due to dilatancy and expansion (see Figure 5(b)).

4.3. Brittle-Ductile Failure of Heterogeneous Soft Coal under Multiple Factor Coupling. After further analysis of the stress-strain failure curves of the specimens with different heterogeneities (shown in Figure 5(a)), the compression process can be divided into the compaction stage, the elastic stage, and the strain-hardening stage. As shown in Figure 5, there is an upwardly convex critical phase between the initial linear elastic compaction phase and the ductile failure phase. And we call this as a critical phase which is a brittle-ductile failure transition phase. The key to distinguish this critical phase is the process of the strain curve from the linear elastic phase to the plastic rupture phase, in which the slope of the tangent line at one point of the overstrain curve is larger than that of the tangent line at the latter point of the critical phase. Figure 6 shows the determination of the critical phase. The slope of the tangent line at point $H_{1}$ is larger than that at point $H_{2}$ (i.e., $\tan \theta_{1}>\tan \theta_{2}$ ), and hence, the slope can be regarded as the critical phase. Several points fit into the critical phase; therefore, in order to determine the critical inflection point $H$ more accurately in the critical phase, we can select the average value of stress increments in the critical phase as the critical inflection point value $H$, i.e., the critical inflection point value $H=\Delta \sigma / 2$.

The deformation process of the coal mass exhibits an ideal elastic to brittle feature before point $H$. After this point $H$, the coal mass undergoes typical strain-hardening plastic flow. If the degree of heterogeneity is higher (i.e., the $m$ value increases from 1 to 6 ), the critical inflection point stress value 


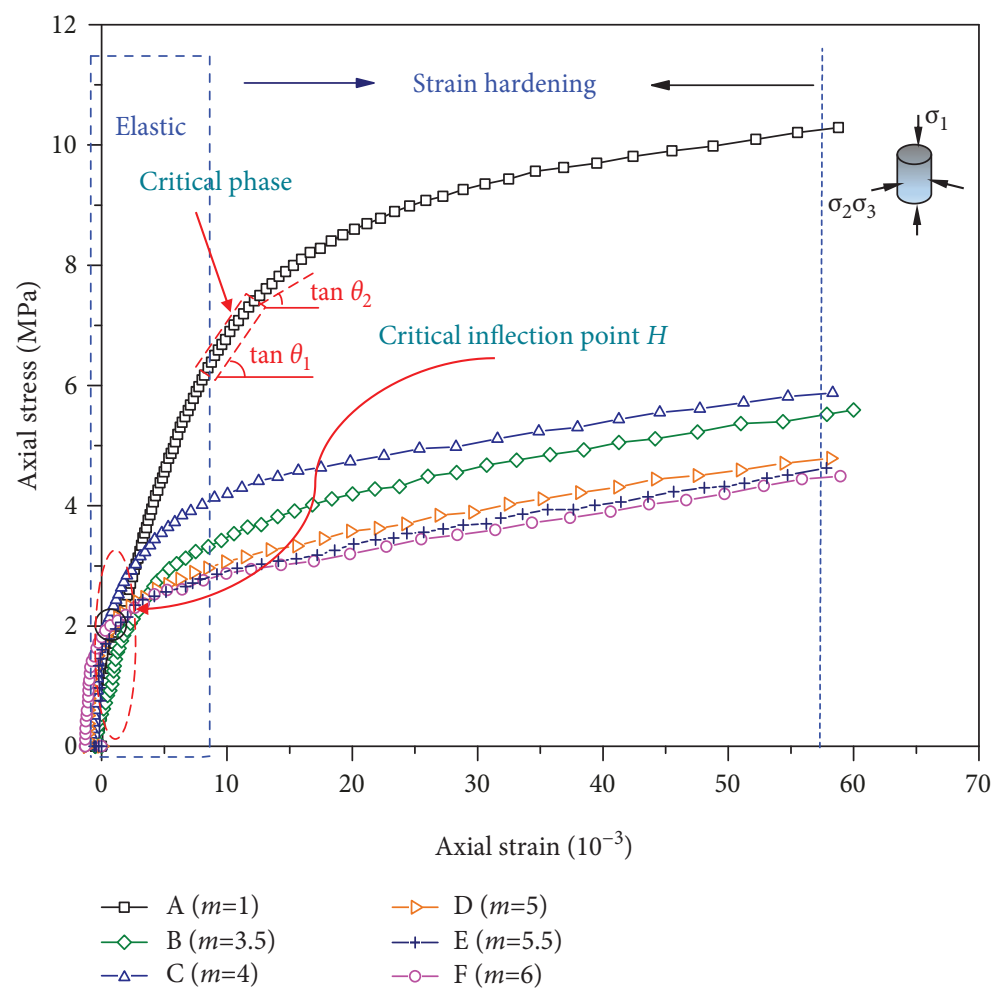

(a)

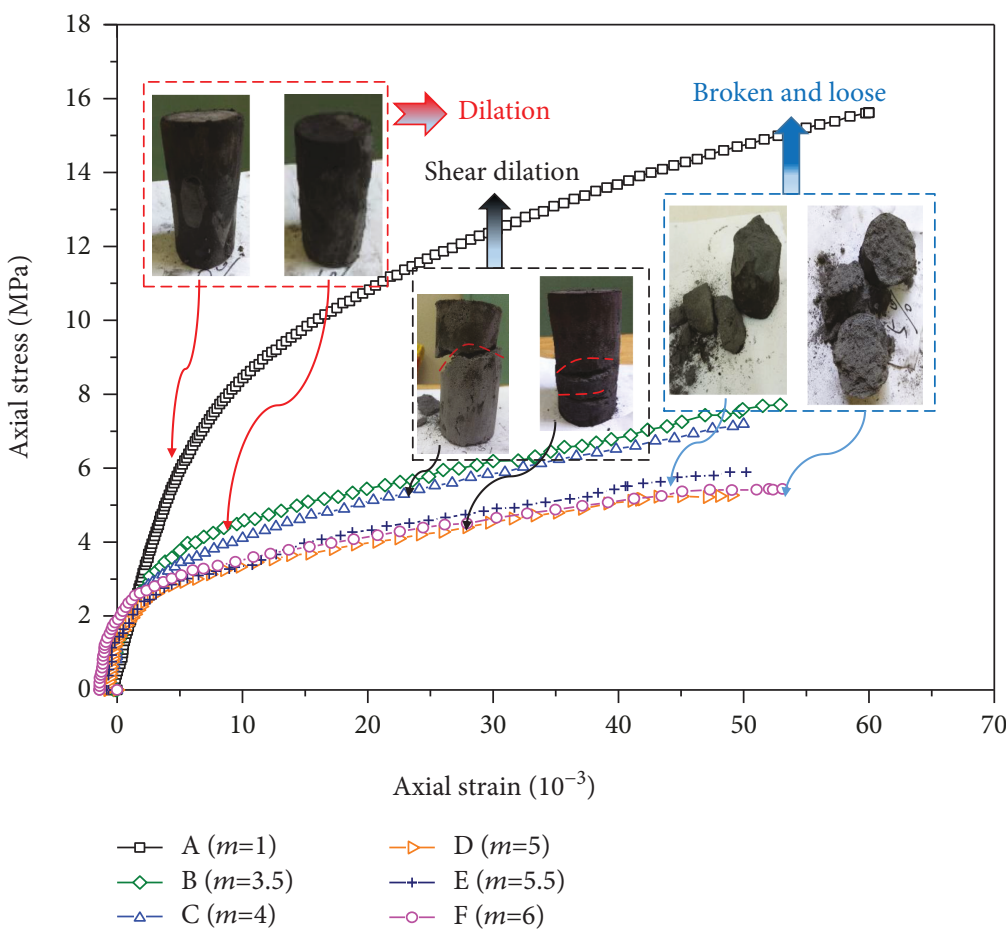

(b)

FiguRE 5: Stress-strain law of the triaxial test under different confining pressures: (a) 2 MPa confining pressure test and (b) 4 MPa confining pressure test results and failure modes of specimens.

$H$ is lower (i.e., the critical inflection point stress value $H$ drops from 7.1 MPa to 2.5 MPa). Besides, the compaction degree of the pore and fracture in the line-elastic phase of the coal sample decreases with the increase of heterogeneity parameter $m$. In contrast, the strain grows rapidly after this point and the stress is almost unchanged. The critical 


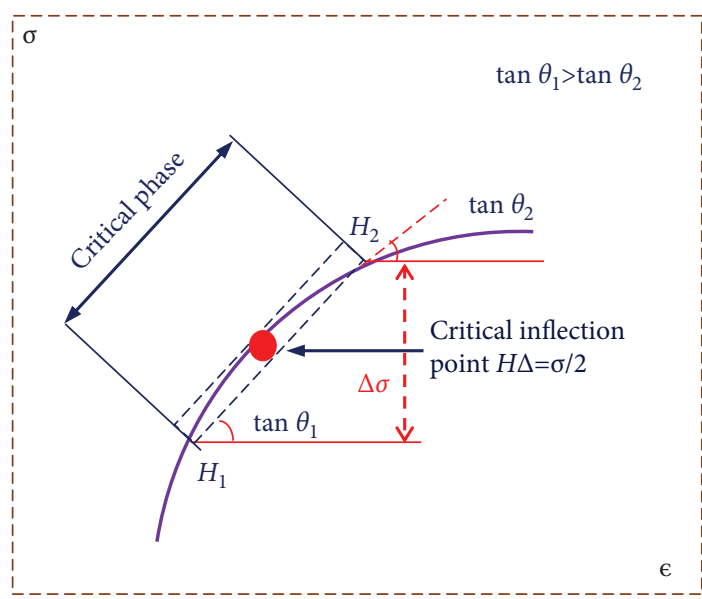

FIGURE 6: Determination of the critical phase and critical inflection point.

inflection point $H$ is one key factor in predicting whether the specimen undergoes ideal brittle failure or the transformation of failure due to strain hardening.

The brittle-ductile transition failure occurs obviously in the soft coal body under the coupling of multiple factors (e.g., confining pressure and heterogeneity). To further explore whether or not the intrinsic factor (heterogeneity) of the soft coal mass can change the brittle-ductile transition failure of the coal, in this section, the critical inflection point stress value $H$ of the brittle-ductile failure transition of the heterogeneous soft coal mass is analyzed statistically, and the relationship between the critical inflection point stress value and the degree of heterogeneity is obtained, as shown in Figure 7.

The linear regression equation of the test data was obtained by linear function regression, as follows:

(i) Under $2 \mathrm{MPa}$ confining pressure:

$$
\begin{aligned}
H & =11.63 m^{-0.897}, \\
R^{2} & =0.939
\end{aligned}
$$

(ii) Under $4 \mathrm{MPa}$ confining pressure:

$$
\begin{aligned}
H & =-0.038 m^{2}-0.105 m+3.891, \\
R^{2} & =0.937
\end{aligned}
$$

Considering fixed external factors (confining pressure), equations (9) and (10) further indicate that the coal mass with a higher degree of heterogeneity $m$ has a lower stress value for the brittle-ductile critical inflection point $H$ and that the probability of brittle-ductile transition of coal increases, i.e., an easier transition from dilatancy and expansion to loose and broken rock. In addition, the variation of the confining pressure has some effect on the change of the

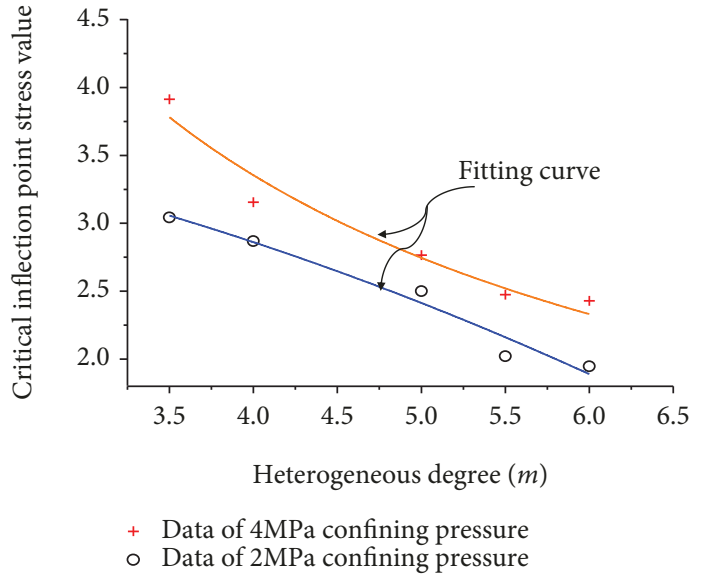

FIgURE 7: The relationship between the critical inflection point stress value and the degree of heterogeneity of the soft coal mass.

critical inflection point stress value. The higher the confining pressure is, the larger the value of $H$. Therefore, a higher confining pressure can increase the integrity of the soft coal mass and enhance its bearing capacity.

\section{Brittle-Ductile Failure Transformation of Soft Coal}

5.1. Brittle-Ductile Failure. The process from brittle failure to ductile failure of rock is not merely the result of a single factor but more the result of multifactor coupling. In actual complex underground rock engineering, the coupling of high pressure, high temperature, and high pressure gas is often encountered. Therefore, the multifactor coupling is the fundamental reason for the brittle-ductile failure transformation of rock. However, it has great limitations with regard to analyzing the brittle-ductile transition of rocks when compared to only using a single factor, which is an idealized assumption of rock mechanics research. The brittle-ductile failure transition of soft coal refers to the three-dimensional stress relief caused by excavation, gas pressure reduction caused by gas drainage from boreholes, increased heterogeneity of coal mass, or the superposition of various engineering blasting and mining disturbance stresses in deep complex rock engineering. These processes change the external factors (stress environment) and internal factors (heterogeneity) of the coal mass, causing the coal mass to undergo a change from stress concentration to stress relief and then to stress concentration and the corresponding rock to undergo a shift from compaction to loose and then to compaction. Therefore, the change refers to the evolution of the coupling factors between the internal homogeneity and the external stress state of the rock, resulting in different degrees of the brittle-ductile transformation of the coal mass.

The distinction between the brittle and the ductile behaviour depends on whether a macroscopic fracture forms (strain localization occurs) after substantial permanent straining (see Figure 8) [44]. In soft coal, brittle deformation is cataclastic in nature, where deformation involves microcrack formation and frictional sliding along grain boundaries, whereas ductile 


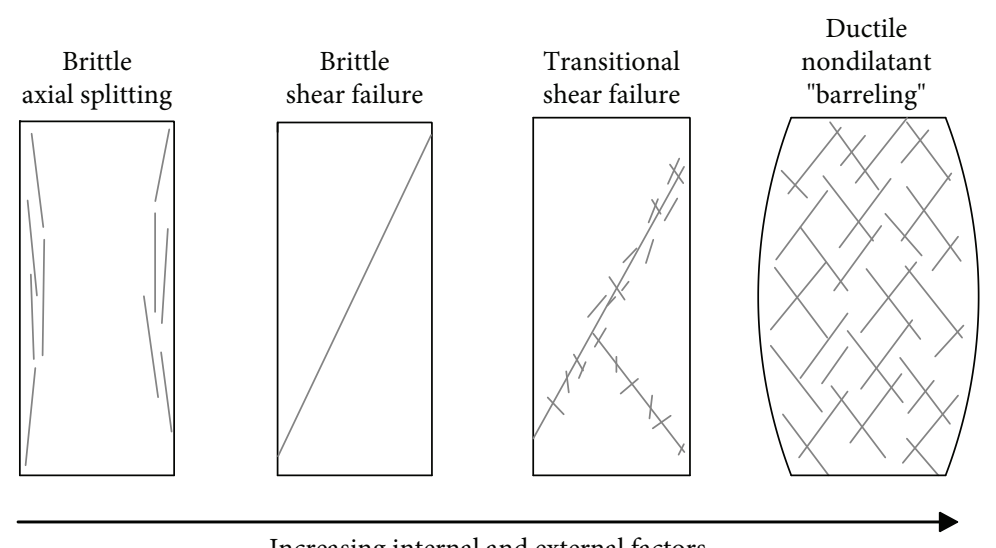

FIGURE 8: Schematic illustrating changes in failure patterns in relation to internal and external factors and ductility (revised from [44]).

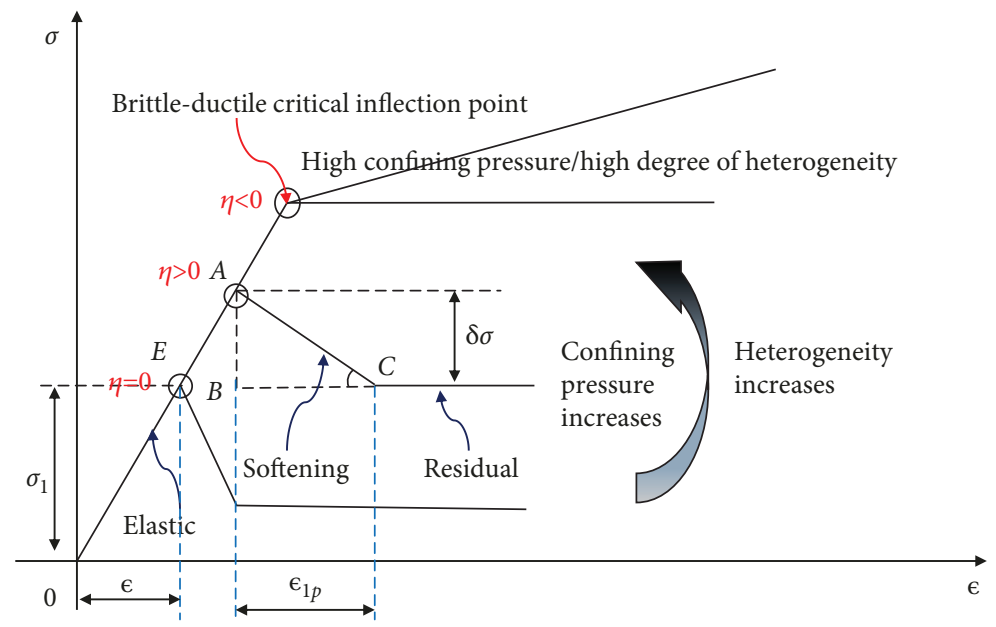

FIGURE 9: Idealized triaxial stress-strain curve of soft coal mass (revised from $[32,55,56]$ ).

deformation (different degrees of heterogeneity) transitions to delocalized cataclasis (or "cataclastic flow"). Also, as deformation becomes increasingly ductile, internal factors (pore collapse) begin to play a more significant role than microcracking, leading to an initially compactant stage in porous soft coal deformation.

By simplifying the curves and data in Figures 5 and 7, the stress-strain curve of the idealized soft coal mass is obtained $[32,55,56]$, as shown in Figure 9. The influence of the confining pressure and the degree of heterogeneity coupling factors on the failure evolution of the soft coal mass is described by $\tan \angle A C B$ in the figure. Under the influence of an external factor, i.e., a low confining pressure, the damage form of the coal mass is an ideal elastic-plastic failure. Both the $\tan \angle A C B$ and the elastic-plastic-ductile transition of the failure state of the coal mass decrease faster due to the increase in the confining pressure. Furthermore, the residual strength of the postpeak coal mass increases, and the ductility is enhanced. The heterogeneity of the soft coal mass also has a significant impact on the transformation between failure modes. The higher the heterogeneity parameters are, the faster the change in the elastic-plastic-ductile failure state of the coal mass (the lower critical inflection point value). According to the results given in Figure 7, the influences of the confining pressure and heterogeneity on the brittle-ductile critical transition state of soft coal are discussed in the next section.

5.2. External Factors (Confining Pressure) on BrittlenessDuctility of Soft Coal Mass. The value of $\tan \angle A C B$ in Figure 9 can be named the brittle modulus, which is the absolute value of the slope of the stress-strain curve at the softening stage after the peak softening of the coal rock specimen and is obtained by

$$
E_{b}=\frac{\delta \sigma}{\varepsilon_{1 p}}
$$

where $\delta \sigma$ is the difference between the peak strength and the residual strength and $\varepsilon_{1 p}$ is the axial plastic strain during the process of coal mass strength degradation.

Figure 9 shows that with an increase in the confining pressure, the postpeak brittleness and the plasticity of soft coal decrease and the transition to ductile damage gradually evolves. During the increase in confining pressure, a 
TABLE 2: Brittle-ductile modulus for different rocks.

(a)

\begin{tabular}{lccccc}
\hline & \multicolumn{3}{c}{ Tennessee marble (data from [55, 57]) } & & \\
\hline Confining pressure $\sigma_{3}(\mathrm{MPa})$ & 0.00 & 3.45 & 6.90 & 13.80 & 20.70 \\
Peak strength $\sigma(\mathrm{MPa})$ & 130 & 145 & 160 & 180 & 195 \\
Residual strength $\sigma_{r}(\mathrm{MPa})$ & 10 & 60 & 80 & 110 & 130 \\
Brittleness modulus $E_{b}(\mathrm{MPa})$ & 174545 & 104615 & 85333 & 74666 & 34666 \\
$\gamma$ & 1.000 & 0.599 & 0.489 & 0.428 & 0.199 \\
\hline
\end{tabular}

(b)

\begin{tabular}{|c|c|c|c|c|c|}
\hline \multicolumn{6}{|c|}{ Gebdykes dolomite (data from [58]) } \\
\hline Confining pressure $\sigma_{3}(\mathrm{MPa})$ & 0 & 5 & 20 & 40 & 90 \\
\hline Peak strength $\sigma(\mathrm{MPa})$ & 60 & 98 & 131 & 150 & 180 \\
\hline Residual strength $\sigma_{r}(\mathrm{MPa})$ & 10 & 42 & 101 & 148 & 200 \\
\hline Brittleness modulus $E_{b}(\mathrm{MPa})$ & 16667 & 8000 & 3529 & 2000 & 1900 \\
\hline$\gamma$ & 1.000 & 0.480 & 0.212 & 0.120 & 0.113 \\
\hline
\end{tabular}

(c)

\begin{tabular}{|c|c|c|c|c|c|}
\hline \multicolumn{6}{|c|}{ Vosges sandstone (data from [59]) } \\
\hline Confining pressure $\sigma_{3}(\mathrm{MPa})$ & 0 & 10 & 20 & 40 & 60 \\
\hline Peak strength $\sigma(\mathrm{MPa})$ & 31 & 74 & 92 & 108 & 110 \\
\hline Residual strength $\sigma_{r}(\mathrm{MPa})$ & 22 & 38 & 75 & 94 & 107 \\
\hline Brittleness modulus $E_{b}(\mathrm{MPa})$ & 6923 & 4500 & 4232 & 3337 & 1060 \\
\hline$\gamma$ & 1.000 & 0.650 & 0.561 & 0.482 & 0.153 \\
\hline
\end{tabular}

(d)

\begin{tabular}{|c|c|c|c|c|}
\hline \multicolumn{5}{|c|}{ Heterogeneous soft coal rock mass $m=1$} \\
\hline Confining pressure $\sigma_{3}(\mathrm{MPa})$ & 0 & 1 & 2 & 4 \\
\hline Peak strength $\sigma$ or critical inflection point value $H(\mathrm{MPa})$ & 4.5 & 6.2 & 7.1 & 8.2 \\
\hline Residual strength $\sigma_{r}$ or ductility strength $(\mathrm{MPa})$ & 2.2 & 8.3 & 10.1 & 15.3 \\
\hline Brittleness modulus $E_{b}(\mathrm{MPa})$ & 575 & 241 & 143 & 70 \\
\hline$\gamma$ & 1.000 & 0.330 & 0.248 & 0.121 \\
\hline
\end{tabular}

dynamically evolving postpeak brittle-ductile modulus factor can be established:

$$
\gamma=\frac{E_{b}}{E_{b 0}}, \quad 0<g<1
$$

where $E_{\mathrm{b} 0}$ is the brittle modulus of the soft coal mass under uniaxial loading, $E_{b 0}=\sigma_{1} / \varepsilon$, and $E_{b}$ is the brittle modulus of the soft coal mass under confining pressure.

The corresponding brittle modulus and brittleness coefficient obtained from the triaxial test data of Tennessee marble $[55,57]$, Gebdykes dolomite [58], Vosges sandstone [59], and heterogeneous soft coal mass are shown in Table 2, and the relationship between the confining pressure and the brittleductile modulus of the rock is shown in Figure 10. The brittle modulus $E_{\mathrm{b}}$ of the rock changes gradually with the confining pressure; i.e., the brittle-ductile modulus coefficient $\gamma$ of the rock undergoes an evolution process with the change of the confining pressure (the $\gamma$ value changes from 0 to 1 ). According to the heterogeneous coal rock mass and multiple experimental $[55,57-59]$ results, we obtained the variation law of the brittle-ductile transition inflection point stress $H$ of the rock (see Figure 6). Based on this law, in the relation curve of the confining pressure and brittle-ductile modulus, a brittle-ductile modulus transition boundary of rock can be determined. At present, the brittle-ductile modulus $\gamma$ value is approximately 0.2. According to Figure 10, from the analysis, it can be seen that when the $\gamma$ value is between 0.2 and 1 , the coal and rock mass show ideal brittle failure, and the corresponding brittle modulus coefficient is now called the 


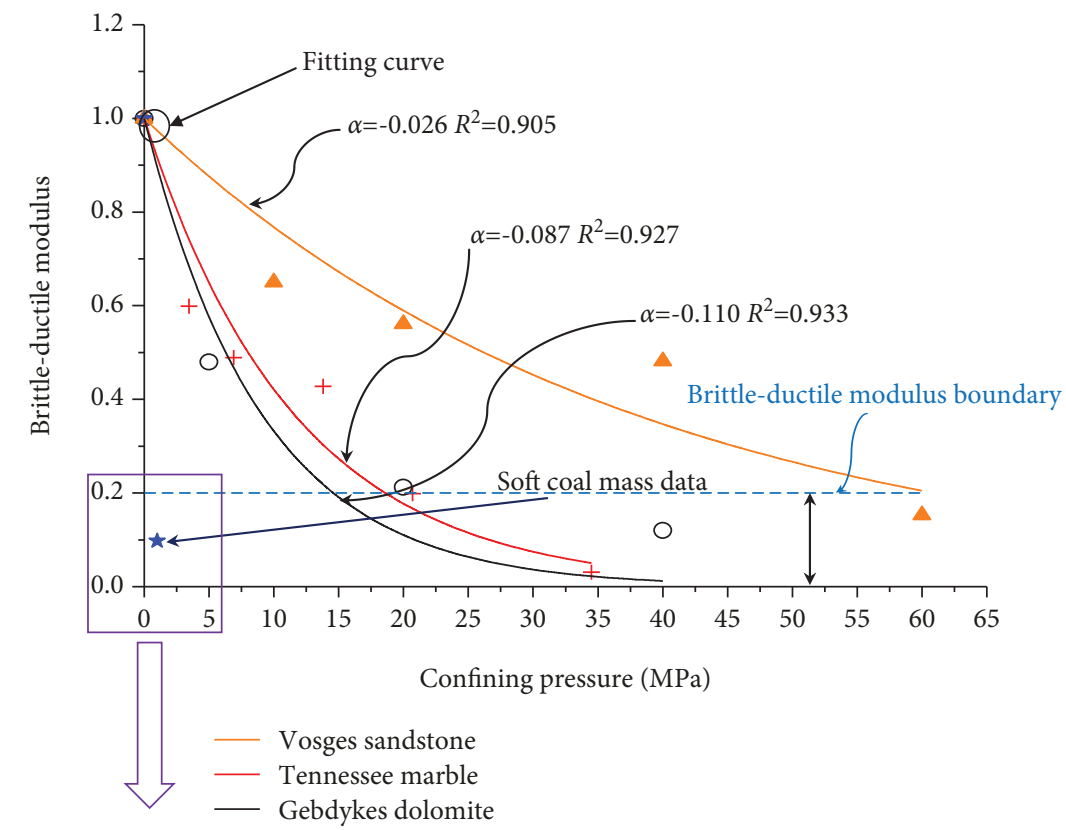

(a)

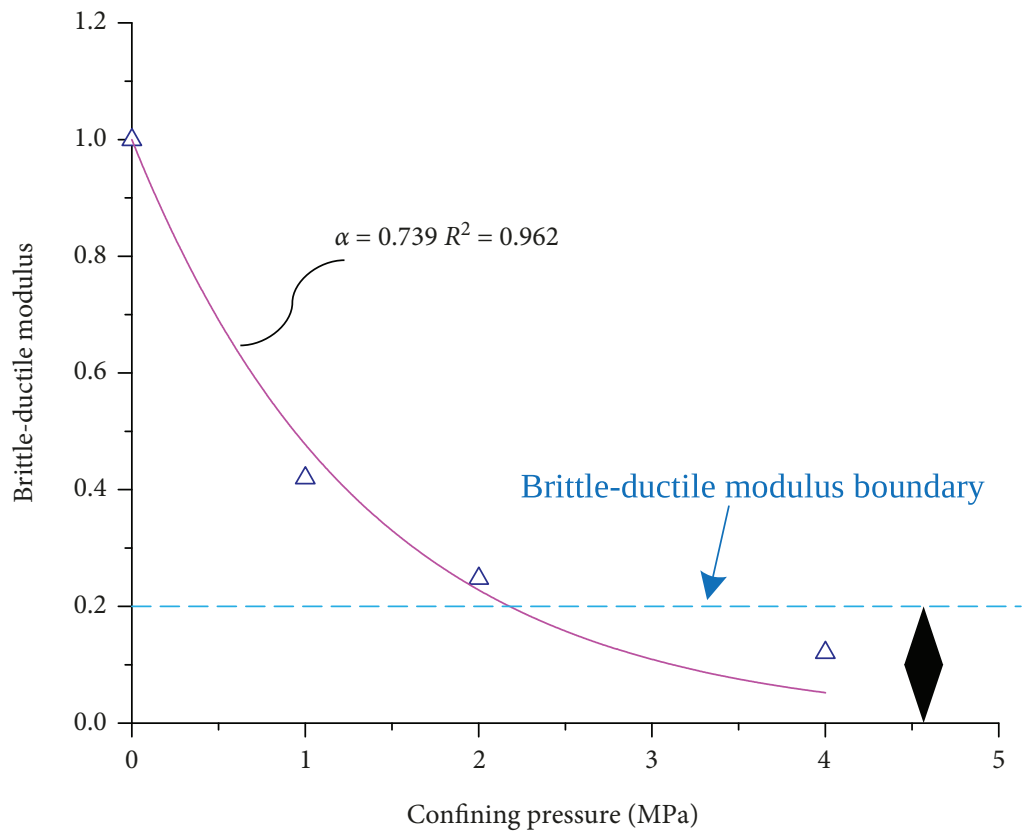

$\triangle$ Shoft coal

_ Fitting curve

(b)

FIGURE 10: The relationship between confining pressure and rock brittle-ductile modulus: (a) brittle rock; (b) heterogeneous soft coal rock mass.

brittle modulus coefficient (Figure 10(b)). When the confining pressure continues to increase, $\gamma$ decreases gradually from 0.2 infinitely to 0 . Then, the coal mass transitions from brittle to ductile failure, and the mechanical characteristics are similar to those of the soft rock. The corresponding brittle-ductile modulus coefficient is called the ductile modulus coefficient with a small value (i.e., $\gamma$ is within $0 \sim 0.2$ ).
If this kind of soft coal mass is under high pressure, regardless of the damage characteristics or mechanical properties of the coal, ductile failure flow appears under the condition of a small confining pressure. Therefore, according to the test results, the modulus coefficient of heterogeneous soft coal is generally in the range of 0 to 0.2 .

By fitting the test data, the relationship between the brittle-ductile modulus coefficient $\gamma$ and the confining 


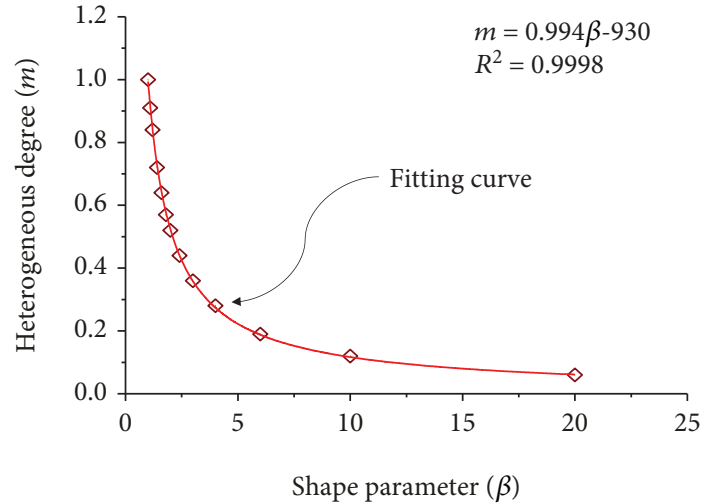

FIGURE 11: The relationship between the shape parameter $\beta$ and the inhomogeneous parameter $m$.

pressure almost obeys a negative exponential function. With further increases in the confining pressure, the brittle-ductile modulus coefficient $\gamma$ decreases to a certain extent, and the following relationship can be obtained [60]:

$$
\gamma=\frac{E_{b}}{E_{b 0}}=\exp \left(-a \sigma_{3}\right)
$$

where $a$ is the fitting constant of the experiment and $\sigma_{3}$ is the confining pressure.

5.3. Internal Factor (Heterogeneity Degree Parameter) on the Brittleness-Ductility of Soft Coal Mass. Whether brittle failure or ductile-plastic failure occurs in the coal mass depends on not only the stress environment but also the impact tendency of the coal mass. The impact tendency of the coal mass is also closely related to the heterogeneity parameter $m$ of the coal mass. Hence, the relationship between the brittle-ductile state transitions of soft coal rock is described by introducing the index $\eta$ of the impact tendency as follows:

$$
\eta=\frac{E}{E_{b}}
$$

where $E$ is the elastic modulus before the peak strength of the coal mass and $E_{b}$ is the brittle modulus of the coal and rock under a certain confining pressure.

Similarly, as shown in Figure 9, the range of values represents the physical and mechanical properties of the coal mass, with $\eta=\infty$ representing the ideal elastoplastic material, $\eta=0$ representing the extremely brittle material, $\eta<0$ representing the strain-hardening material, and $0<\eta<\infty$ representing the softening material.

The shape parameter $\beta$ of the Weibull distribution indicates the heterogeneity parameter $m$ of the mesoelement [61]. The relationship between the shape parameter $\beta$ and the heterogeneous parameter $m$ can be obtained from the fitting curve of the soft coal mass, as shown in Figure 11.
The equation of the shape parameter $\beta$ and the heterogeneous parameter $m$ can be obtained by fitting the experimental data curve, as follows:

$$
m=0.994 \beta^{-0.93},
$$

where $\beta$ is the shape parameter.

The above relations can be described as follows.

$$
\eta=\frac{E}{E_{b}}=b(\beta-1)^{-c},
$$

where both $b$ and $c$ are material constants.

Based on the above analysis, with an increase in the shape parameter $\beta$ (i.e., $\beta$ is increased from 0 to 20 ), the heterogeneity parameter $m$ of the coal mass decreases gradually (i.e., the $m$ value is reduced from 1 to infinitely close to 0 ). A decrease in the heterogeneity parameter $m$ causes the impact tendency index $\eta$ to decrease gradually (see Figure 11). Meanwhile, if the brittleness of the coal mass increases, the critical softening depth of the surrounding rock will decrease, and the rock will be prone to dynamic phenomena, e.g., coal and gas outburst. According to the change law of the critical brittle-ductile inflection point of soft coal with different heterogeneities (see Figure 7), the higher the heterogeneity parameter $m$ is, the smaller the stress intensity of the brittle-ductile critical inflection point of the coal mass and the more obvious the ductile failure characteristic stage. In contrast, the lower the heterogeneity parameter $m$ of the soft coal rock is, the higher the homogeneity of the coal mass and the more homogeneous the mineral composition structure. Therefore, more ideal brittle fracture characteristics of the coal mass are reflected in the macromechanical characteristics.

Equation (17) can be obtained from equations (13) and (16) as follows:

$$
\frac{E}{E_{b 0}}=B(\beta-1)^{-c},
$$

where $B=\exp \left(-a \sigma_{3}\right) \cdot b$.

The material parameter $b$ in the expression of the impact tendency index $\eta$ of the soft coal mass is related to the confining pressure. If the confining pressure is not considered, i.e., $\sigma_{3}=0$, equation (17) is consistent with equation (16). At the same time, the brittle-ductile transition relationship of the soft coal and rock is related only to the heterogeneity parameter $m$. Therefore, even if only considering the heterogeneity of soft coal, it also plays a vital role in the brittle-ductile transition failure of soft coal. If the confining pressure is increased, the coupling effect of the confining pressure and heterogeneity parameter $m$ influences the damage to the coal body. With a change in the confining pressure and the heterogeneity parameter $m$, both the factors have an influence on the change rate of the shock tendency. Both the heterogeneity $m$ of the coal mass and the confining pressure coupling not only determine the speed of the transition between the brittle and the ductile failure of the coal rock but also affect 
(a)

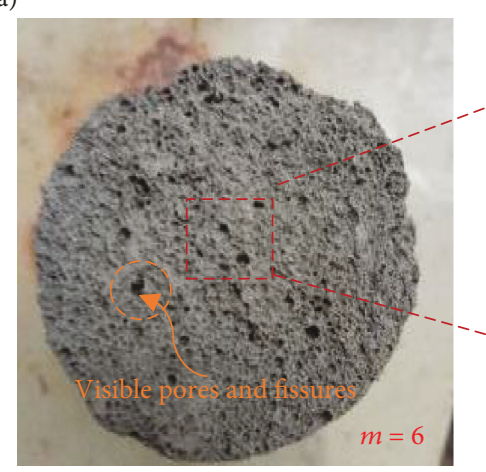

(b)

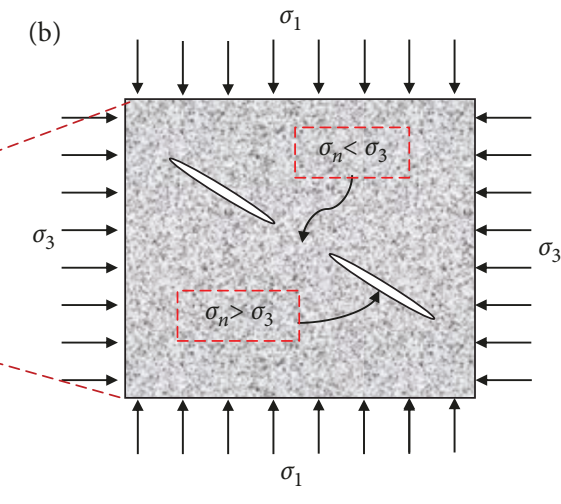

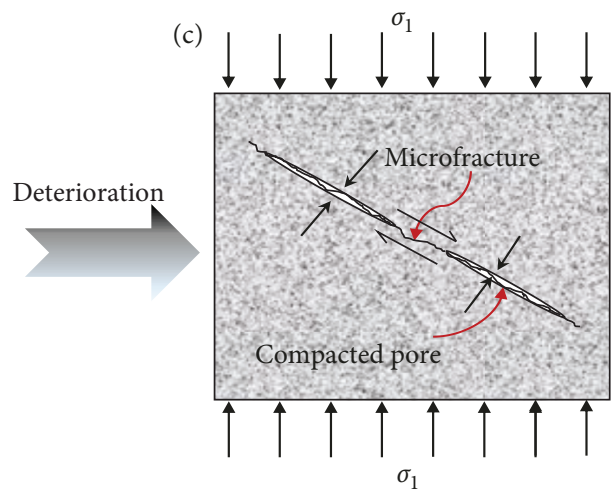

FIgURE 12: Microfracture propagation in a soft coal mass due to a macrofailure process. (a) Fracture diagram of the coal mass specimen $(m=6)$. (b) Three-dimensional stress stability structure of the coal mass. (c) The microfracture develops into a macroscopic fissure.

the general failure mode of the gas-bearing coal mass and the probability of coal and gas outburst and rock burst tendency in the underground roadway.

Figure 12 illustrates the evolution of microfracture propagation relative to the fracture macroscopic failure process in the coal rock mass with high heterogeneity $(m=6)$. The soft coal mass forms an initial porosity with different degrees after the internal gas drainage. From a mesoscopic point of view, the effective normal stress $\sigma_{n}$ is less than the macroconfining pressure $\sigma_{3}$, but the effective normal stress $\sigma_{n}$ of the joint is greater than the confining pressure $\sigma_{3}$ of the coal mass (see Figure 12(b)), so the ability of the microfissure to resist macroshear yielding of the coal is limited [62]. When the lateral unloading yield of the coal mass is shifted from the three-direction stress state to the two-direction unbalanced stress concentration, the microcrack tends to open, and the stress development deterioration causes a new coalescence fracture between the original fissures. When the lateral limit is large, the whole surface will produce a smooth shear fracture, but when the lateral limit is small, the surface will produce a rough split crack (see Figure 12(c)).

\section{Conclusions}

This study attempts a new experimental research idea, based on the heterogeneity characteristics of the soft coal mass, by using hydrogen peroxide $\left(\mathrm{H}_{2} \mathrm{O}_{2}\right)$ in an alkaline medium to generate oxygen as the major impact indicators. Different proportioning schemes and different kinds of heterogeneous soft coal mass specimens were made in cast-iron moulds. A series of multifactor coupling mechanical tests were carried out for soft coal masses with different heterogeneities, and the brittle-ductile response of a soft coal rock mass was discussed. The following conclusions can be drawn.

(1) The uniaxial and triaxial compression tests were carried out on heterogeneous soft coal samples. The ductility failure characteristic is reflected; i.e., the test curve has no apparent peak strain and residual strength, which presents a kind of similarity to the rheology of soft rock flow. The coal specimens in the loading process show internal factors (different degrees of heterogeneity $m$ ) that vary due to the degradation effect on the coal mass, from coal body crack initiation and propagation, overlapping with the process of heterogeneous coal mass destruction from shear compression to bulging expansion and even fracture failure

(2) In the heterogeneous mechanical test curve of the soft coal specimen, there is an upwardly convex critical phase between the initial linear elastic compaction phase and the ductile failure phase. A key point can be determined at this phase which is called the critical inflection point $H$ of the brittle-ductile failure transition. Before this point, ideal elastoplastic characteristics are expressed. In contrast, after this point or phase, the mechanical properties of the coal masses are transformed into ductile strain hardening

(3) The more the confining pressure and the degree of the heterogeneity parameter $m$ increase, the more obvious the ductile failure of the specimens is. The heterogeneity degree of rock is also the critical factor for the brittle-ductile transition of rocks

(4) With the influence of coal mass excavation or gas drainage, the coal rock mass meso- and macropore and fracture development will expand, weaken the strength of the coal mass, or even cause the deterioration of the internal structure, resulting in the incomplete integrity of the coal mass and, after shear and dilatancy, the evolution from loose to broken rock

\section{Data Availability}

The data used to support the findings of this study are available from the corresponding authors upon request.

\section{Conflicts of Interest}

The authors declare that they have no conflicts of interest. 


\section{Acknowledgments}

This study was supported by the National Natural Science Foundation of China (51574122, 51434006, and 51774130). The financial support is greatly appreciated. We also appreciate the reviewers and editors for their careful work and thoughtful suggestions that have helped improve this paper substantially. A special acknowledgment is given to $\mathrm{Mr}$. Qingjun Guan and Miss Meichen Du (Hunan University of Science and Technology), who provided language editing for this paper.

\section{References}

[1] S. C. Blair and N. G. W. Cook, "Analysis of compressive fracture in rock using statistical techniques: part I. A nonlinear rule-based model," International Journal of Rock Mechanics and Mining Sciences, vol. 35, no. 7, pp. 837-848, 1998.

[2] G. Z. Li, C. A. Tang, and L. C. Li, “Three-dimensional micro flow-stress-damage (FSD) model and application in hydraulic fracturing in brittle and heterogeneous rocks," Key Engineering Materials, vol. 452-453, pp. 581-584, 2010.

[3] M. Sabri, A. Ghazvinian, and H. R. Nejati, "Effect of particle size heterogeneity on fracture toughness and failure mechanism of rocks," International Journal of Rock Mechanics and Mining Sciences, vol. 81, pp. 79-85, 2016.

[4] C. H. Scholz, "Microfracturing and the inelastic deformation of rock in compression," Journal of Geophysical Research, vol. 73, no. 4, pp. 1417-1432, 1968.

[5] S. C. Yuan and J. P. Harrison, "A review of the state of the art in modelling progressive mechanical breakdown and associated fluid flow in intact heterogeneous rocks," International Journal of Rock Mechanics and Mining Sciences, vol. 43, no. 7, pp. 1001-1022, 2006.

[6] C. H. Zhang, Q. S. Zhao, and Y. J. Yu, "Model of coupled gas flow and deformation process in heterogeneous coal seams and its application," Journal of Coal Science and Engineering (China), vol. 17, no. 1, pp. 76-80, 2011.

[7] W. C. Zhu, J. Liu, T. H. Yang, J. C. Sheng, and D. Elsworth, "Effects of local rock heterogeneities on the hydromechanics of fractured rocks using a digital-image-based technique," International Journal of Rock Mechanics and Mining Sciences, vol. 43, no. 8, pp. 1182-1199, 2006.

[8] T. K. Lu, Z. J. Zhao, and H. F. Hu, "Improving the gate road development rate and reducing outburst occurrences using the waterjet technique in high gas content outburst-prone soft coal seam," International Journal of Rock Mechanics and Mining Sciences, vol. 48, no. 8, pp. 1271-1282, 2011.

[9] M. Mastalerz, A. Drobniak, D. Strąpoć, W. Solano Acosta, and J. Rupp, "Variations in pore characteristics in high volatile bituminous coals: implications for coal bed gas content," International Journal of Coal Geology, vol. 76, no. 3, pp. 205-216, 2008.

[10] G. Wang, W. X. Li, P. F. Wang, X. X. Yang, and S. T. Zhang, "Deformation and gas flow characteristics of coal-like materials under triaxial stress conditions," International Journal of Rock Mechanics and Mining Sciences, vol. 91, pp. 72-80, 2017.

[11] S. G. Wang, D. Elsworth, and J. S. Liu, "Permeability evolution in fractured coal: the roles of fracture geometry and water- content," International Journal of Coal Geology, vol. 87, no. 1, pp. 13-25, 2011.

[12] R. Q. Huang and X. N. Wang, "Analysis of dynamic disturbance on rock burst," Bulletin of Engineering Geology and the Environment, vol. 57, no. 3, pp. 281-284, 1999.

[13] M. Krzesinska, "Ultrasonic studies of outburst-prone coals," International Journal of Rock Mechanics and Mining Sciences, vol. 34, no. 1, pp. 167-171, 1997.

[14] J. Shepherd, L. K. Rixon, and L. Griffiths, "Outbursts and geological structures in coal mines: a review," International Journal of Rock Mechanics and Mining Sciences \& Geomechanics Abstracts, vol. 18, no. 4, pp. 267-283, 1981.

[15] S. P. Singh, "Burst energy release index," Rock Mechanics and Rock Engineering, vol. 21, no. 2, pp. 149-155, 1988.

[16] N. Skoczylas, "Laboratory study of the phenomenon of methane and coal outburst," International Journal of Rock Mechanics and Mining Sciences, vol. 55, pp. 102-107, 2012.

[17] J. Toraño, S. Torno, E. Alvarez, and P. Riesgo, “Application of outburst risk indices in the underground coal mines by sublevel caving," International Journal of Rock Mechanics and Mining Sciences, vol. 50, pp. 94-101, 2012.

[18] Y. Xue, F. Gao, T. Teng, and Y. Xing, "Effect of gas pressure on rock burst proneness indexes and energy dissipation of coal samples," Geotechnical and Geological Engineering, vol. 34, no. 6, pp. 1737-1748, 2016.

[19] A. Zhou, K. Wang, L. Li, and C. Wang, "A roadway driving technique for preventing coal and gas outbursts in deep coal mines," Environmental Earth Sciences, vol. 76, no. 6, p. 236, 2017.

[20] A. Zubelewicz and Z. Mróz, "Numerical simulation of rock burst processes treated as problems of dynamic instability," Rock Mechanics and Rock Engineering, vol. 16, no. 4, pp. 253-274, 1983.

[21] S. C. Li and G. Y. Li, "Effect of heterogeneity on mechanical and acoustic emission characteristics of rock specimen," Journal of Central South University of Technology, vol. 17, no. 5, pp. 1119-1124, 2010.

[22] P. Z. Pan, X. T. Feng, and J. A. Hudson, "The influence of the intermediate principal stress on rock failure behaviour: a numerical study," Engineering Geology, vol. 124, pp. 109-118, 2012.

[23] J. Song and K. Kim, "Micromechanical modeling of the dynamic fracture process during rock blasting," International Journal of Rock Mechanics and Mining Sciences \& Geomechanics Abstracts, vol. 33, no. 4, pp. 387-394, 1996.

[24] S. H. Wang, C. I. Lee, P. G. Ranjith, and C. A. Tang, "Modeling the effects of heterogeneity and anisotropy on the excavation damaged/disturbed zone (EDZ)," Rock Mechanics and Rock Engineering, vol. 42, no. 2, pp. 229-258, 2009.

[25] P. K. Kaiser and C. A. Tang, "Numerical simulation of damage accumulation and seismic energy release during brittle rock failure-part II: rib pillar collapse," International Journal of Rock Mechanics and Mining Sciences, vol. 35, no. 2, pp. 123134, 1998.

[26] C. A. Tang and P. K. Kaiser, "Numerical simulation of cumulative damage and seismic energy release during brittle rock failure-part I: fundamentals," International Journal of Rock Mechanics and Mining Sciences, vol. 35, no. 2, pp. 113-121, 1998.

[27] L. Biolzi, J. F. Labuz, and G. Muciaccia, “A problem of scaling in fracture of damaged rock," International Journal of Rock 
Mechanics and Mining Sciences, vol. 48, no. 3, pp. 451-457, 2011.

[28] Y. L. Tan, D. M. Huang, and Z. Zhang, "Rock mechanical property influenced by inhomogeneity," Advances in Materials Science and Engineering, vol. 2012, Article ID 418729, 9 pages, 2012.

[29] W. J. Yu and F. F. Liu, "Stability of close chambers surrounding rock in deep and comprehensive control technology," Advances in Civil Engineering, vol. 2018, Article ID 6275941, 18 pages, 2018.

[30] G. S. Wu, W. J. Yu, Z. Liu, and Z. Tang, "Failure law and mechanism of the rock-loose coal composite specimen under combined loading rate," Advances in Civil Engineering, vol. 2018, Article ID 2482903, 10 pages, 2018.

[31] Q. J. Guan, W. Sun, Y. Hu et al., "Simultaneous control of particle size and morphology of $\alpha$-CaSO $4 \cdot 1 / 2 \mathrm{H}_{2} \mathrm{O}$ with organic additives," Journal of the American Ceramic Society, vol. 102, pp. 2440-2450, 2018.

[32] Z. C. Feng and Y. S. Zhao, "Correlativity of rock inhomogeneity and rock burst trend," Chinese Journal of Rock Mechanics and Engineering, vol. 11, pp. 1863-1865, 2003.

[33] Y. F. Yang, C. A. Tang, and K. W. Xia, "Study on crack curving and branching mechanism in quasi-brittle materials under dynamic biaxial loading," International Journal of Fracture, vol. 177, no. 1, pp. 53-72, 2012.

[34] N. Hudyma, B. B. Avar, and M. Karakouzian, "Compressive strength and failure modes of lithophysae-rich Topopah Spring tuff specimens and analog models containing cavities," Engineering Geology, vol. 73, no. 1-2, pp. 179-190, 2004.

[35] G. Ma, Y. D. Zhang, W. Zhou, T. T. Ng, Q. Wang, and X. Chen, "The effect of different fracture mechanisms on impact fragmentation of brittle heterogeneous solid," International Journal of Impact Engineering, vol. 113, pp. 132-143, 2018.

[36] B. Liang, M. Zhang, Y. S. Pan, and Y. J. Wang, "The experimental research on the effect of gas on mechanical properties and mechanical response of coal," Chinese Journal of Geotechnical Engineering, vol. 17, pp. 12-18, 1995.

[37] G. Z. Yin, M. H. Li, J. G. Wang, J. Xu, and W. P. Li, "Mechanical behavior and permeability evolution of gas infiltrated coals during protective layer mining," International Journal of Rock Mechanics and Mining Sciences, vol. 80, pp. 292-301, 2015.

[38] B. Wu, T. Ma, G. Feng, Z. Chen, and X. Zhang, "An approximate solution for predicting the heat extraction and preventing heat loss from a closed-loop geothermal reservoir," Geofluids, vol. 2017, Article ID 2041072, 17 pages, 2017.

[39] B. Wu, X. Zhang, R. G. Jeffrey, A. P. Bunger, and C. Huddlestone-Holmes, "Perturbation analysis for predicting the temperatures of water flowing through multiple parallel fractures in a rock mass," International Journal of Rock Mechanics and Mining Sciences, vol. 76, pp. 162-173, 2015.

[40] S. Q. Yang, M. Chen, H. W. Jing, K. F. Chen, and B. Meng, "A case study on large deformation failure mechanism of deep soft rock roadway in Xin'an coal mine, China," Engineering Geology, vol. 217, pp. 89-101, 2017.

[41] W. J. Yu, W. J. Wang, G. S. Wu, X. Y. Yu, and W. Q. Peng, "Three zones and support technique for large section incline shaft crossing goaf," Geotechnical and Geological Engineering, vol. 35, no. 5, pp. 1921-1931, 2017.
[42] W. J. Yu, S. H. Zhai, and Q. Gao, "Stability evaluation indexes of deep stope pillar and roadway surrounding rock," Disaster Advances, vol. 5, no. 4, pp. 120-126, 2012.

[43] K. Hu, Q. Z. Zhu, L. Chen, J. F. Shao, and J. Liu, "A micromechanics-based elastoplastic damage model for rocks with a brittle-ductile transition in mechanical response," Rock Mechanics and Rock Engineering, vol. 51, no. 6, pp. 1729-1737, 2018.

[44] G. Walton, A. Hedayat, E. Kim, and D. Labrie, "Post-yield strength and dilatancy evolution across the brittle-ductile transition in Indiana limestone," Rock Mechanics and Rock Engineering, vol. 50, no. 7, pp. 1691-1710, 2017.

[45] Q. H. Wu, L. Weng, Y. L. Zhao, B. Guo, and T. Luo, "On the tensile mechanical characteristics of fine-grained granite after heating/cooling treatments with different cooling rates," Engineering Geology, vol. 253, pp. 94-110, 2019.

[46] G. S. Wu, W. J. Yu, P. Wang, Z. Liu, F. F. Liu, and Z. Huan, "Deformation failure mechanism and experimental study of gas coal rock mass based on percolation mechanism," Journal of China Coal Society, vol. 43, no. 3, pp. 724-734, 2018.

[47] Z. Zhou, Y. Y. Lu, Z. L. Ge, F. Yang, and X. W. Zhang, "Theoretical and experimental study on strength characteristics of coal under coupling effect of water and gas," Journal of China Coal Society, vol. 39, no. 12, pp. 2419-2424, 2014.

[48] J. Bodziony, J. Krawczyk, and J. Topolnicki, “Determination of the porosity distribution in coal briquettes by measurements of the gas filtration parameters in an outburst pipe," International Journal of Rock Mechanics and Mining Sciences \& Geomechanics Abstracts, vol. 31, no. 6, pp. 661669, 1994.

[49] C. B. Jiang, G. Z. Yin, X. Q. Li, and B. Cai, "Experimental study of gas permeability of outburst coal briquettes in complete stress-strain process," Chinese Journal of Rock Mechanics and Engineering, vol. 29, pp. 3482-3487, 2010.

[50] N. Skoczylas, B. Dutka, and J. Sobczyk, "Mechanical and gaseous properties of coal briquettes in terms of outburst risk," Fuel, vol. 134, pp. 45-52, 2014.

[51] Q. H. Niu, L. W. Cao, S. X. Sang, X. Z. Zhou, Z. Z. Wang, and $\mathrm{Z}$. Y. Wu, "The adsorption-swelling and permeability characteristics of natural and reconstituted anthracite coals," Energy, vol. 141, pp. 2206-2217, 2017.

[52] Q. H. Niu, L. W. Cao, S. X. Sang, X. Z. Zhou, and Z. Z. Wang, "Anisotropic adsorption swelling and permeability characteristics with injecting $\mathrm{CO}_{2}$ in coal," Energy and Fuels, vol. 32, no. 2, pp. 1979-1991, 2018.

[53] C. E. Fairhurst and J. A. Hudson, "Draft ISRM suggested method for the complete stress-strain curve for intact rock in uniaxial compression," International Journal of Rock Mechanics and Mining Sciences \& Geomechanics Abstracts, vol. 36, no. 3, pp. 279-289, 1999.

[54] E. T. Brown, Ed., Rock Characterization, Testing \& Monitoring: ISRM Suggested Methods, Pergamon Press, Oxford, UK, 1981.

[55] Z. Fang and J. P. Harrison, "A mechanical degradation index for rock," International Journal of Rock Mechanics and Mining Sciences, vol. 38, no. 8, pp. 1193-1199, 2001.

[56] C. H. Zhang, Q. S. Zhao, L. G. Wang, N. Zhao, and Y. J. Yu, "Test and numerical modeling on strain softening behavior and permeability evolution of rock under tri-axial compression," Journal of China Coal Society, vol. 8, pp. 1774-1782, 2015. 
[57] X. Tan, H. Konietzky, and T. Frühwirt, "Laboratory observation and numerical simulation of permeability evolution during progressive failure of brittle rocks," International Journal of Rock Mechanics and Mining Sciences, vol. 68, pp. 167-176, 2014.

[58] S. C. Yuan and J. P. Harrison, "An empirical dilatancy index for the dilatant deformation of rock," International Journal of Rock Mechanics and Mining Sciences, vol. 41, no. 4, pp. 679-686, 2004.

[59] P. Bésuelle, J. Desrues, and S. Raynaud, "Experimental characterisation of the localisation phenomenon inside a Vosges sandstone in a triaxial cell," International Journal of Rock Mechanics and Mining Sciences, vol. 37, no. 8, pp. 12231237, 2000.

[60] Z. Z. Zhang, F. Gao, and X. J. Shang, "Rock burst proneness prediction by acoustic emission test during rock deformation," Journal of Central South University, vol. 21, no. 1, pp. 373-380, 2014.

[61] R. Luo, Y. W. Zeng, Y. Cao, and L. Li, "Research on influence of inhomogeneity degree on mechanical parameters of inhomogeneous rock," Rock and Soil Mechanics, vol. 33, pp. 3788-3794, 2012.

[62] Q. Jiang, X. T. Feng, and G. Q. Chen, "Study on constitutive model of hard rock considering surrounding rock deterioration under high geostresses," Chinese Journal of Rock Mechanics and Engineering, vol. 27, pp. 144-152, 2008. 

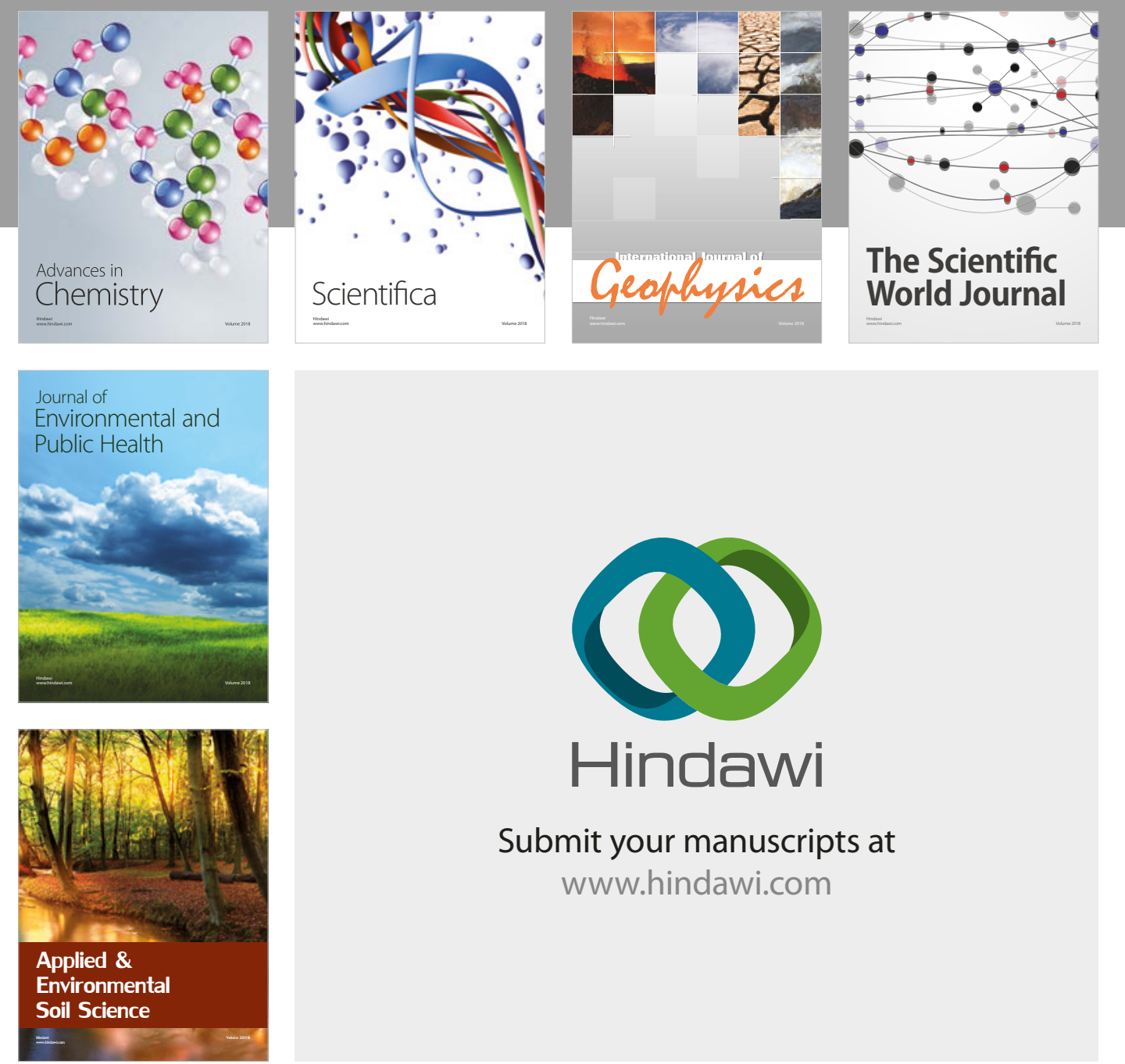

The Scientific

\section{World Journal}
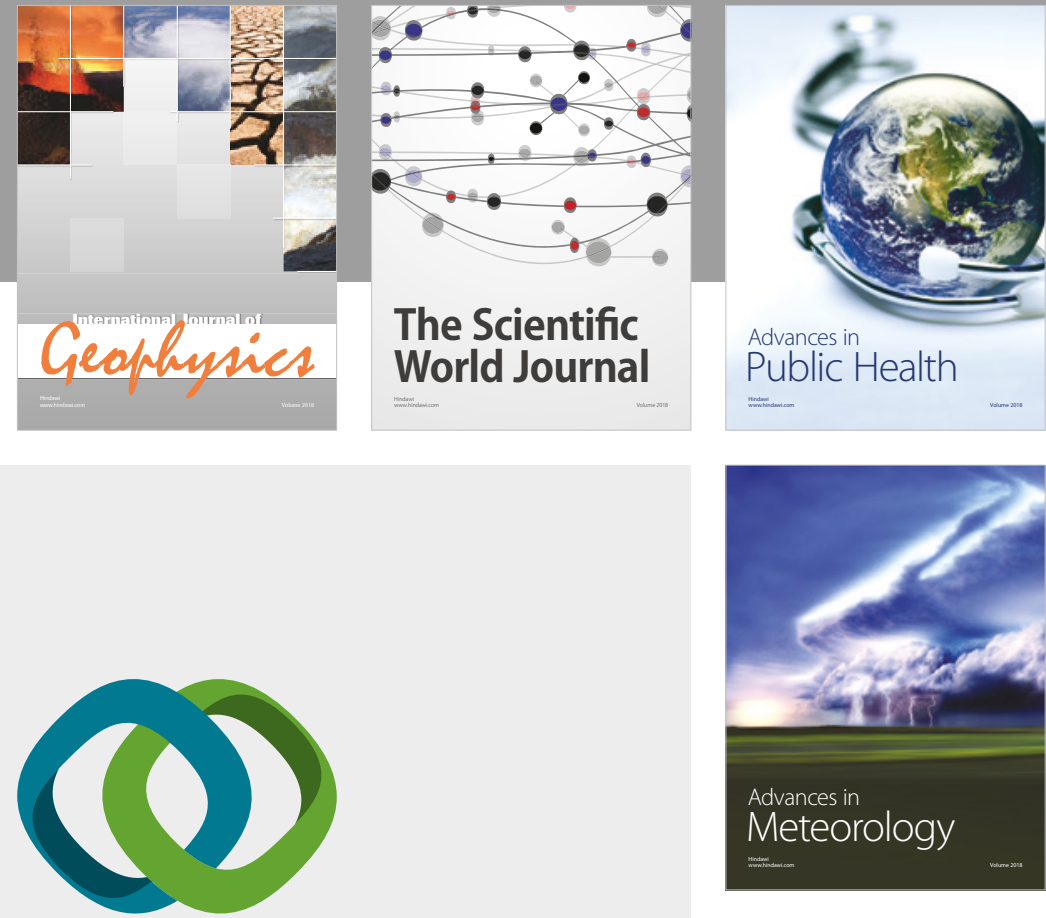

Advan

Public Health

\section{Hindawi}

Submit your manuscripts at

www.hindawi.com
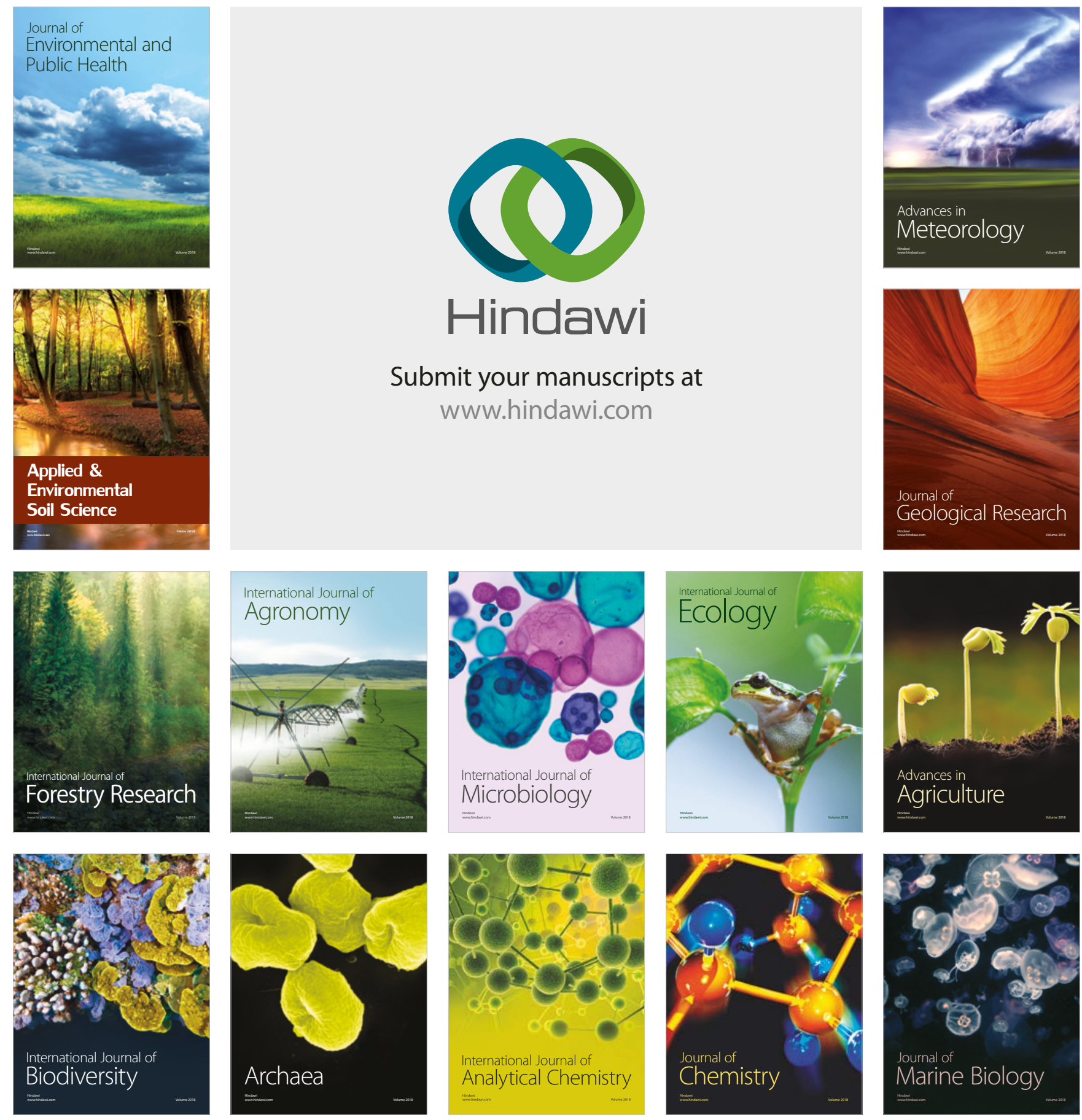